\title{
ON THE INTERSECTION OF A CLASS OF MAXIMAL SUBGROUPS OF A FINITE GROUP
}

\author{
GUO XIUYUN
}

(Communicated by Warren J. Wong)

\begin{abstract}
Let $G$ be a finite group and $\pi$ a set of primes. We consider the family of subgroups of $G: \mathscr{F}=\left\{M: M<\cdot G,[G: M]_{\pi}=1,[G: M]\right.$ is composite $\}$ and denote $S_{\pi}(G)=\bigcap\{M: M \in \mathscr{F}\}$ if $\mathscr{F}$ is non-empty, otherwise $S_{\pi}(G)=G$. The purpose of this note is to prove

Theorem. Let $G$ be a $\pi$-solvable group. Then $S_{\pi}(G)$ has the following properties: (1) $S_{\pi}(G) / O_{\pi}(G)$ is supersolvable. (2) $S_{\pi}\left(S_{\pi}(G)\right)=S_{\pi}(G)$. (3) $G / O_{\pi}(G)$ is supersolvable if and only if $S_{\pi}(G)=G$.
\end{abstract}

There has been much interest in the past in considering various generalisations of the Frattini subgroup of a finite group and to investigate the influence of such a subgroup on the structure of the group (see, Deskins [1], Gaschütz [2], Rose [3], and [4]). In [4], P. Bhattacharya and N. P. Mukherjee introduce a subgroup $S_{\pi}(G)$ and exhibit its relationship with the given group $G$. The objective of this paper is to investigate the subgroup $S_{\pi}(G)$ further. We obtain the following result.

Theorem. Let $G$ be a finite $\pi$-solvable group, then $S_{\pi}(G) / O_{\pi}(G)$ is a supersolvable group.

Let $\pi$ be any set of primes and $\pi^{\prime}$ the complementary set of primes. Let $G$ be a finite group. Then we denote $M<\cdot G$ to indicate that $M$ is a maximal subgroup of $G$. Also, $[G: M]_{\pi}$ denotes the $\pi$-part of $[G: M]$. Now consider the following family of subgroups:

$$
\mathscr{F}=\left\{M: M<\cdot G,[G: M]_{\pi}=1,[G: M] \text { is composite }\right\} .
$$

Definition. $S_{\pi}(G)=\bigcap\{M: M \in \mathscr{F}\}$ if $\mathscr{F}$ is non-empty, otherwise $S_{\pi}(G)=$ $G$. Clearly, $S_{\pi}(G) \unlhd G, \Phi(G) \leq S_{\pi}(G)$ and $O_{\pi}(G) \leq S_{\pi}(G)$, where $\Phi(G)$ is the Frattini subgroup of the group $G$ and $O_{\pi}(G)$ is the maximal normal $\pi$-subgroup of $G$.

All groups in this paper are finite.

Received by the editors September 22, 1988.

1980 Mathematics Subject Classification (1985 Revision). Primary 20D20.

Key words and phrases. Finite group, maximal subgroup. 
Lemma 1. Let $H$ be a solvable normal subgroup of a group $G, 1 \neq N \leq H$, $N \unlhd G$, and let $H / N$ be a supersolvable group. Then $G=N \cdot N_{G}(V)$, where $V$ is a supersolvable projector of $H$ and is not normal in $G$.

Proof. By [5, Theorem 5, 1, 7] a supersolvable projector $V$ of $H$ exists, and $H=N V$. Consequently $H=N V^{g}$ for every $g \in G$ since $H \unlhd G$. It is proved easily that $V^{g}$ is a supersolvable projector of $H$ also. Thus, there is $h \in H$ such that $V^{g}=V^{h}$, and $g \in N_{G}(V) \cdot H$. The result now follows.

Lemma 2. If $G$ is a $\pi$-solvable group, then $S_{\pi}(G)$ is a solvable group.

Proof. We assume that $S_{\pi}(G) \neq 1$. Let $N$ be a minimal normal subgroup of $G$ with $N \leq S_{\pi}(G)$. Then by induction hypothesis $S_{\pi}(G) / N$ is solvable because $S_{\pi}(G / N)=S_{\pi}(G) / N$ ([4, Lemma 1]). Since $G$ is $\pi$-solvable, we have that $N$ is either a $\pi^{\prime}$-group or a $p$-group for some prime $p \in \pi$. If $N$ is a $p$-group, then $S_{\pi}(G)$ is solvable. If $N$ is a $\pi^{\prime}$-group, then $N$ is contained in every $\pi^{\prime}$-Hall subgroup of $G$ and $N \leq S_{\pi^{\prime}}(G)$. Consequently, $N \leq S_{\pi}(G) \cap S_{\pi^{\prime}}(G)$. By [4, Lemma 2] and [4, Theorem 3] $S_{\pi}(G) \cap S_{\pi^{\prime}}(G)$ is supersolvable and $N$ is solvable. Thus, $S_{\pi}(G)$ is a solvable group. The Lemma is proved.

Proof of Theorem. Since $O_{\pi}\left(G / O_{\pi}(G)\right)=1$ and $S_{\pi}\left(G / O_{\pi}(G)\right)=S_{\pi}(G) / O_{\pi}(G)$, we may assume that $O_{\pi}(G)=1$ and $S_{\pi}(G) \neq 1$.

Case 1. $G$ has a minimal normal subgroup $N \leq S_{\pi}(G)$ such that $O_{\pi}(G / N)=$ 1 . By induction hypothesis $S_{\pi}(G) / N$ is supersolvable. Let $V$ be a supersolvable projector of $S_{\pi}(G)$. Then $S_{\pi}(G)=N V$.

If $V \unlhd G$, then $V \cap N \not G$. Since $N$ is a minimal normal subgroup $N \cap V=1$ or $N$. Consequently, $S_{\pi}(G)=N \times V$ or $V$. Notice that $V$ is a supersolvable projector of $S_{\pi}(G)$. Thus, $S_{\pi}(G)=V$ is supersolvable.

If $V \unlhd G$, then by Lemma 1 there is a maximal subgroup $M$ of $G$ such that $N_{G}(V) \leq M$ and $G=N M$. Notice that $N$ is an abelian $p$-group for some prime $p \in \pi^{\prime}$; thus $M \cap N=1$ and $[G: M]_{\pi}=1$. If $[G: M]$ is composite, then $N \leq S_{\pi}(G) \leq M$ and $G=M$, contradiction. Thus, [G:M] is a prime and $N$ is a cyclic group of prime order. Consequently $S_{\pi}(G)$ is supersolvable.

Case 2. For every minimal normal subgroup $N\left(\leq S_{\pi}(G)\right)$ of $G, O_{\pi}(G / N) \neq 1$.

Let $N \leq S_{\pi}(G)$ be a minimal normal subgroup of $G,|N|=p^{\alpha}, p \in \pi^{\prime}$, and let $T / N \leq S_{\pi}(G) / N$ be a minimal normal $\pi$-subgroup of $G / N,|T / N|=q^{\beta}$ for some prime $q \in \pi$. If $Q \in \operatorname{Syl}_{q} T$, then by a Frattini argument $G=$ $N \cdot N_{G}(Q)$. Since $Q \nsubseteq G$, there is a maximal subgroup $M$ of $G$ such that $N_{G}(Q) \leq M$ and $G=N M$. It is obvious that $N \$ \Phi(G)$. Since $N$ is a minimal normal subgroup $M \cap N=1,[G: M]_{\pi}=1$. Consequently, $[G: M]$ is a prime and $N$ is a cyclic group of prime order. Thus, we have proved the following:

(1) $N$ is a cyclic group of prime order for every minimal normal subgroup $N\left(\leq S_{\pi}(G)\right)$ of $G$.

(2) $\Phi(G)=1$. 
(3) The fitting subgroup $F\left(S_{\pi}(G)\right)$ of $S_{\pi}(G)$ is the direct product of minimal normal subgroups of $G$ which are contained in $S_{\pi}(G)$.

In fact, let $H$ be maximal among all subgroups of $F\left(S_{\pi}(G)\right)$ which can be expressed as the direct product of minimal normal subgroups of $G$. Note then that $H \unlhd G$. We will first show that there is a subgroup $S$ of $G$ such that $G=H S$ and $H \cap S=1$.

Let $S$ be a minimal element of set $\{T: H T=G, T \leq G\}$. Then, $S \cap$ $H \unlhd G(S \cap H \unlhd S$ because $H \unlhd G ; S \cap H \unlhd H$ because $H$ is abelian; hence $S \cap H \unlhd S H=G$ ). If $S \cap H \neq 1$, then since $\Phi(G)=1$, there is a maximal subgroup $M$ of $G$ such that $G=M(S \cap H)$. We now see that $S=S \cap G=(S \cap M)(S \cap H)$ and, since $M$ does not contain $S \cap H$, that $S \cap M<S$. However, $G=S H=(S \cap M)(S \cap H) H=(S \cap M) H$. This contradicts the choice of $S$ and we conclude that $S \cap H=1$.

We next consider the subgroup $S \cap F\left(S_{\pi}(G)\right)$. It is normal in both $S$ and in $F\left(S_{\pi}(G)\right.$ ) (the latter because $\Phi(G)=1$ implies $\Phi\left(F\left(S_{\pi}(G)\right)\right)=1$, but $\left[F\left(S_{\pi}(G)\right)\right]^{\prime} \leq \Phi\left(F\left(S_{\pi}(G)\right)\right)$ since $F\left(S_{\pi}(G)\right)$ is nilpotent, hence $F\left(S_{\pi}(G)\right)$ is abelian). Thus, it is normal in $G$. If $S \cap F\left(S_{\pi}(G)\right) \neq 1$, then there is a minimal normal subgroup $N$ of $G$ with $N \leq S \cap F\left(S_{\pi}(G)\right)$. As $S \cap H=1$, we conclude that $H<N \times H$. This contradicts the choice of $H$ and therefore $S \cap F\left(S_{\pi}(G)\right)=1$. It now follows that $H=F\left(S_{\pi}(G)\right)$ and the result is proved.

(4) $S_{\pi}(G)$ is supersolvable.

Let $M$ be a maximal subgroup of $S_{\pi}(G)$. If $M \geq F\left(S_{\pi}(G)\right)$, then $M$ does not contain some minimal normal subgroup $N$ of $G$ which is contained in $S_{\pi}(G)$ by (3). Hence $S_{\pi}(G)=M N$ and $M \cap N=1$. By (1), $N$ is a cyclic group of prime order and therefore $\left[S_{\pi}(G): M\right]$ is a prime. Since $\left[S_{\pi}(G): M\right]=$ [ $\left.F\left(S_{\pi}(G)\right): F\left(S_{\pi}(G)\right) \cap M\right]$, it follows that $M \cap F\left(S_{\pi}(G)\right)$ is a maximal subgroup of $F\left(S_{\pi}(G)\right)$. By [5, Theorem $\left.1,3,3\right] S_{\pi}(G)$ is a supersolvable group.

Corollary 1. Let $G$ be a $\pi$-solvable group. Then a $\pi^{\prime}$-Hall subgroup $H$ of $S_{\pi}(G)$ is supersolvable.

Corollary 2. Let $G$ be a $\pi$-solvable group. Then $S_{\pi}\left(S_{\pi}(G)\right)=S_{\pi}(G)$.

Proof. Since $S_{\pi}(G) / O_{\pi}(G)$ is supersolvable and $O_{\pi}(G)$ is contained in every $\pi$-Hall subgroup of $S_{\pi}(G)$, every maximal subgroup of $S_{\pi}(G)$ which contains a $\pi$-Hall subgroup of $S_{\pi}(G)$ is of prime index in $S_{\pi}(G)$, or there is no maximal subgroup which contains a $\pi$-Hall subgroup of $S_{\pi}(G)$ in $S_{\pi}(G)$. Hence $S_{\pi}\left(S_{\pi}(G)\right)=S_{\pi}(G)$.

Corollary 3. Let $G$ be a $\pi$-solvable group. Then $G / O_{\pi}(G)$ is supersolvable if and only if $G / S_{\pi}(G)$ is supersolvable.

Proof. Since $O_{\pi}(G) \leq S_{\pi}(G)$, the necessity is obvious. Let $G / S_{\pi}(G)$ be supersolvable. Then $S_{\pi}\left(G / S_{\pi}(G)\right)=G / S_{\pi}(G)$, but by [4, Lemma 1] $S_{\pi}\left(G / S_{\pi}(G)\right)=$ $S_{\pi}(G) / S_{\pi}(G)=1$, therefore, $S_{\pi}(G)=G$ and $G / O_{\pi}(G)=S_{\pi}(G) / O_{\pi}(G)$ is supersolvable by the theorem.

It is obvious that Corollary 3 is equivalent to 
Corollary 4. If $G$ is $\pi$-solvable, then $G / O_{\pi}(G)$ is supersolvable if and only if $S_{\pi}(G)=G$.

\section{REFERENCES}

1. W. E. Deskins, On maximal subgroups, First Sympos. Pure Math., Amer. Math. Soc. Providence, RI, 1959.

2. W. Gaschütz, Über die Untergruppe endlicher Gruppen, Math. Z. 58 (1953), 260-270.

3. J. Rose, The influence on a group of its abnormal structure, J. Math. Lond. Soc. 40 (1965), 348-361.

4. P. Bhattacharya and N. P. Mukherjee, On the intersection of a class of maximal subgroups of a finite group II, J. Pure and Applied Algebra (North-Holland) 42 (1986), 117-124.

5. M. Weistein, Between nilpotent and solvable, Polygonal Publishing House, 1982.

Department of Mathematics, Shanxi University, Taiyuan, China 